\title{
ON THE DISTORTION OF $n$-DIMENSIONAL QUASICONFORMAL MAPPINGS
}

\author{
MATTI VUORINEN
}

\begin{abstract}
A new upper bound $c(n, K)$ for the linear dilatation of a $K$-quasiconformal mapping of a domain in $R^{n}$ is proved. This upper bound substantially improves the previously known $n$-dimensional bound and it is asymptotically sharp when $n=2$.
\end{abstract}

1. Introduction. In this paper we shall prove some theorems about the distortion of quasiconformal mappings defined on the $n$-dimensional Möbius space $\bar{R}^{n}=R^{n} U$ $\{\infty\}, n \geqslant 2$, equipped with the spherical metric $q$,

$$
\begin{aligned}
& q(x, y)=|x-y|\left(1+|x|^{2}\right)^{-1 / 2}\left(1+|y|^{2}\right)^{-1 / 2} ; \quad x \neq \infty \neq y, \\
& q(x, \infty)=\left(1+|x|^{2}\right)^{-1 / 2} ; \quad x \in R^{n} .
\end{aligned}
$$

Besides the spherical metric we shall often require the Poincare metric of $R_{+}^{n}=$ $\left\{\left(x_{1}, \ldots, x_{n}\right) \in R^{n}: x_{n}>0\right\}$ denoted by $\rho$ or $\rho_{R_{+}^{n}}$.

In his recent book, A. F. Beardon [B, p. 42] proved the following theorem which yields the sharp Lipschitz constant of a Möbius transformation in the spherical metric.

1.2. THEOREM. If $f: \bar{R}^{n} \rightarrow \bar{R}^{n}$ is a Möbius transformation and $f^{*}: \bar{R}^{n+1} \rightarrow \bar{R}^{n+1}$ is its Poincaré extension with $f^{*} R_{+}^{n+1}=R_{+}^{n+1}$, then

$$
\operatorname{Lip}(f)=\sup _{x \neq y} \frac{q(f(x), f(y))}{q(x, y)}=\exp \rho_{R_{+}^{n+1}}\left(e_{n+1}, f^{*}\left(e_{n+1}\right)\right)
$$

We shall extend this result to the case of quasiconformal mappings. Since quasiconformal mappings are locally Hölder continuous with an exponent which may be less than one, the above supremum may be infinite in the case of these mappings. In order to circumvent this technical difficulty we introduce

$$
\delta_{f}=\inf \{q(x, y): q(f(x), f(y)) \geqslant 1 / 2\} \in[0,1]
$$

whenever $f: \bar{R}^{n} \rightarrow \bar{R}^{n}$ is a continuous mapping. Then, if $f$ is a Möbius transformation,

$$
\operatorname{Lip}(f) \leqslant 1 / \delta_{f} \leqslant 2 \operatorname{Lip}(f)
$$

Received by the editors July 13, 1984 and, in revised form, February 22, 1985.

1980 Mathematics Subject Classification. Primary 30C60.

Key words and phrases. Quasiconformal mappings. 
(see Corollary 2.12). Assume now that $f: \bar{R}^{n} \rightarrow \bar{R}^{n}$ is a $K$-quasiconformal mapping and let $f^{*}: \bar{R}^{n+1} \rightarrow \bar{R}^{n+1}$ be its $K^{*}$-quasiconformal extension with $K^{*}=K^{*}(n, K)$ (see Tukia and Väisälä [TV]). We shall prove the following quasiconformal version of Theorem 1.2.

1.4. THEOREM. If $f: \bar{R}^{n} \rightarrow \bar{R}^{n}$ is a K-quasiconformal mapping, $f^{*}: \bar{R}^{n+1} \rightarrow \bar{R}^{n+1}$ is its extension as above and $\delta_{f}$ is as in (1.3), then

(1) $q(f(x), f(y)) \leqslant\left(\lambda_{n} / \sqrt{3}\right)\left(q(x, y) / \delta_{f}\right)^{\alpha}$, where $\alpha=K^{1 /(1-n)}$ and $\lambda_{n}$ is a number depending only on $n$.

Moreover,

(2) $a_{1} \leqslant \exp \rho_{R_{+}^{n+1}}\left(e_{n+1}, f^{*}\left(e_{n+1}\right)\right) \leqslant a_{2}$, where $a_{1}$ and $a_{2}$ are positive numbers depending only on $n, K^{*}$, and $\delta_{f}$.

Let $G$ be a domain in $R^{n}$ and let $f: G \rightarrow R^{n}$ be a continuous mapping. The linear dilatation of $f$ at $x \in G$ is defined as

$$
H(x, f)=\limsup _{r \rightarrow 0} \frac{L(x, f, r)}{l(x, f, r)},
$$

where for $r \in(0, d(x, \hat{o} G))$,

$$
\begin{aligned}
& L(x, f, r)=\max \{|f(z)-f(x)|:|z-x|=r\}, \\
& l(x, f, r)=\min \{|f(z)-f(x)|:|z-x|=r\} .
\end{aligned}
$$

The main result of this paper is

1.5. TheOREM. Let $G$ be a domain in $R^{n}$ and let $f: G \rightarrow f G \subset R^{n}$ be a K-quasiconformal mapping. Then

$$
H(x, f) \leqslant 1+\tau^{-1}(\tau(1) / K)=c(n, K),
$$

where $\tau(s)$ stands for the capacity of the Teichmüller ring in $R^{n}$.

For $n=2$, A. Mori [M, pp. 61-62] proved a result similar to Theorem 1.5 and his result was generalized to the higher dimensional case by F. W. Gehring [G1, p. 371], however, with a constant $d(n, K)>c(n, K)$. For $n=2$ the best possible constant $\lambda(K)$ in Theorem 1.5 was found by O. Lehto, K. I. Virtanen and J. Väisälä [LVV, p. 8]. The constant $c(n, K)$ in (1.6) seems to be the smallest constant applicable to all dimensions $n \geqslant 2$. In the particular case $n=2$ it is asymptotically sharp, i.e. $c(2, K) / \lambda(K) \rightarrow 1$ as $K \rightarrow \infty$ while $d(2, K) / \lambda(K) \rightarrow 16$ as $K \rightarrow \infty$ (see (3.4)-(3.11)). Several other inequalities relating $c(n, K), d(n, K)$ and $\lambda(K)$ to each other will be proved elsewhere [AVV]. By [AVV], $10 c(n, K)<d(n, K)$ for $n \geqslant 2$.

Finally, we remark that Theorem 1.5 can be immediately extended to the case of quasiregular mappings in $R^{n}, n \geqslant 2$ (see Remark 3.2 below). As a result, one obtains an upper bound for the linear dilatation of these mappings, which improves the previously known bound due to Yu. G. Reshetnyak [R, Theorem 1, p. 1312] and O. Martio, S. Rickman and J. Väisälä [MRV, Theorem 4.5, p. 19].

2. Preliminary results. We shall adopt the relatively standard notation and terminology of [V]. The coordinate unit vectors in $R^{n}$ are $e_{1}, \ldots, e_{n}$. If $x, y \in R^{n}$, then we denote $[x, y]=\{t x+(1-t) y: 0 \leqslant t \leqslant 1\}$ and similarly for open or 
half-open segments. If $x \in R^{n} \backslash\{0\}$, then $[x, \infty)=\{u x: u \geqslant 1\}$. For $x \in R^{n}$ and $r>0$ let $B^{n}(x, r)=\left\{z \in R^{n}: \quad|x-z|<r\right\}, \quad S^{n-1}(x, r)=\partial B^{n}(x, r), \quad B^{n}(r)=$ $B^{n}(0, r), S^{n-1}(r)=\partial B^{n}(r), B^{n}=B^{n}(1)$, and $S^{n-1}=\partial B^{n}$.

For the definition and some properties of the modulus $M(\Delta)$ of a curve family $\Delta$ the reader is referred to $[\mathbf{G 3}, \mathbf{V}]$. If $E, F, G$ are subsets of $R^{n}$ or $\bar{R}^{n}$, then $\Delta(E, F ; G)$ stands for the family of all curves joining $E$ to $F$ in $G$ (see [V, p. 21]). If $G=R^{n}$ or $G=\bar{R}^{n}$ we denote $\Delta(E, F ; G)=\Delta(E, F)$. If $G$ and $G^{\prime}$ are domains in $R^{n}$ or $\bar{R}^{n}, E, F \subset G$, and if $f: G \rightarrow G^{\prime}$ is a $K$-quasiconformal mapping, then $f \Delta(E, F ; G)=\Delta\left(f E, f F ; G^{\prime}\right)$ and (cf. [V, 13.1])

$$
M(\Delta(E, F ; G)) / K \leqslant M\left(\Delta\left(f E, f F ; G^{\prime}\right)\right) \leqslant K M(\Delta(E, F ; G)) .
$$

A ring in $\bar{R}^{n}$ is a domain such that its complement has exactly two components. By definition, the complementary components of the Teichmüller ring $R_{T}(s)$ are $\left[-e_{1}, 0\right]$ and $\left[s e_{1}, \infty\right), s \in(0, \infty)$, while those of the Grötzsch ring $R_{G}(s)$ are $\bar{B}^{n}$ and $\left[s e_{1}, \infty\right), s \in(1, \infty)$. The capacities of these rings, denoted by $\operatorname{cap} R_{T}(s)$ and cap $R_{G}(s)$ respectively, are strictly decreasing functions of $s$. Some properties of cap $R_{T}(s)$ are given in [G3]. A basic formula is

$$
\operatorname{cap} R_{G}(t)=2^{n-1} \operatorname{cap} R_{T}\left(t^{2}-1\right)
$$

for $t \in(1, \infty)$. We denote $\tau(s)=\operatorname{cap} R_{T}(s)$. The well-known equality $\tau(s)=$ $M\left(\Delta\left(\left[-e_{1}, 0\right],\left[s e_{1}, \infty\right)\right)\right)$ will often be used.

2.3. LEMMA. Let $G$ be a domain in $R^{n}$ with $0, x, y \in G$ and assume $0<|x|<|y|<$ $d(0, \partial G) / 2$. If $\tau(s)=\operatorname{cap} R_{T}(s)$, then

(1) $M(\Delta([0, x],[y, \infty) ; G)) \leqslant \tau(|y| /|x|-1)$.

If $E$ and $F$ are continua with $0, x \in E \subset \bar{B}^{n}(|x|), y \in F, F \cap \partial G \neq \varnothing$, then

(2) $M(\Delta(E, F ; G)) \geqslant \tau(|y| /|x|)-2 \omega_{n-1}(\log (d(0, \partial G) /|x|))^{1-n}$.

Proof. Part (1) is a recent result due to F. W. Gehring and it will be published elsewhere [Vu2, 2.58].

To prove part (2) perform a spherical symmetrization of $E$ and $F$ in the positive and negative $x_{1}$-axis, respectively, with center at 0 . Denote the symmetrized sets by $E^{*}, F^{*}$. Then by [G3, Theorem 3, p. 225],

$$
M\left(\Delta\left(E, F ; R^{n}\right)\right) \geqslant M\left(\Delta\left(E^{*}, F^{*} ; R^{n}\right)\right) .
$$

Also by $[\mathbf{V}, 6.2,7.5]$,

$$
\begin{aligned}
& M\left(\Delta\left(E^{*}, F^{*} ; R^{n}\right)\right)+\omega_{n-1}\left(\log \frac{d(0, \partial G)}{|x|}\right)^{1-n} \geqslant \tau(|y| /|x|), \\
& M(\Delta(E, F ; G))+\omega_{n-1}\left(\log \frac{d(0, \partial G)}{|x|}\right)^{1-n} \geqslant M\left(\Delta\left(E, F ; R^{n}\right)\right) .
\end{aligned}
$$

In combination, these inequalities yield the desired lower bound (2).

2.4. Möbius transformations of $B^{n}$. As shown in [A, p. 24] (see also [B, pp. 36-42]) a sense-preserving Möbius transformation $T_{a}: \bar{R}^{n} \rightarrow \bar{R}^{n}$ with $T_{a} B^{n}=B^{n}$ and $T_{a}(a)$ $=0$, where $a \in B^{n}$ is a prescribed point, can be constructed as follows. If $a=0$, set 
$T_{a}=I$ (identity). If $a \in B^{n} \backslash\{0\}$, define $T_{a}(x)=\left(p_{a} \circ \sigma_{a}\right)(x)$, where $\sigma_{a}$ is an inversion in the sphere $S^{n-1}\left(a /|a|^{2}, r\right), r=\sqrt{|a|^{-2}-1}$, orthogonal to $S^{n-1}$, i.e.

$$
\sigma_{a}(x)=a^{*}+r^{2}\left(x-a^{*}\right)^{*}, \quad u^{*}=u /|u|^{2}
$$

and $p_{a}$ is a reflection in the $(n-1)$-dimensional plane orthogonal to $a$ containing 0 . Conversely, a prescribed Möbius transformation $g: \bar{R}^{n} \rightarrow \bar{R}^{n}$ with $g B^{n}=B^{n}$ has a canonical representation [A, p.24; B, pp. 36-42]

$$
g=k \circ T_{a} ; \quad k \in O(n), a \in B^{n},
$$

where $O(n)$ is the set of all orthogonal mappings. We shall employ the Poincaré metric $\rho$ of $B^{n}$ defined by the element of length $d \rho=2|d x| /\left(1-|x|^{2}\right)$. The Poincaré metric of the halfspace $R_{+}^{n}$ is defined by $d \rho=|d x| / x_{n}$.

2.7. Möbius transformations of $\bar{R}^{n}$. We shall identify $\bar{R}^{n}$ with the Riemann sphere $S^{n}\left(e_{n+1} / 2,1 / 2\right) \subset R^{n+1}$ via the stereographic projection $\pi: \bar{R}^{n} \rightarrow S^{n}\left(e_{n+1} / 2,1 / 2\right)$,

$$
\pi(x)=e_{n+1}+\left(x-e_{n+1}\right)^{*} .
$$

The stereographic projection can be used to define the spherical (chordal) metric $q$, see (1.1), by the formula $q(x, y)=|\pi(x)-\pi(y)|$ for $x, y \in \bar{R}^{n}$. For $x \in \bar{R}^{n}$ we define $\tilde{x} \in \bar{R}^{n}$ such that $\pi(x)$ and $\pi(\tilde{x})$ are antipodal (diametrically opposite) points on the Riemann sphere, i.e. $\tilde{0}=\infty, \tilde{\infty}=0$, and $\tilde{x}=-x /|x|^{2}$ for $x \in$ $R^{n} \backslash\{0\}$. For $x \in \bar{R}^{n}, r \in(0,1)$, let $Q(x, r)=\left\{z \in \bar{R}^{n}: q(z, x)<r\right\}$. By virtue of the Pythagorean theorem $Q(x, r)=\bar{R}^{n} \backslash \bar{Q}\left(\tilde{x}, \sqrt{1-r^{2}}\right)$. Hence $\pi Q(x, 1 / \sqrt{2})$ is a hemisphere of the Riemann sphere, $x \in \bar{R}^{n}$. We call a Möbius transformation preserving all spherical distances a spherical isometry. We denote by $h_{z}: \bar{R}^{n} \rightarrow \bar{R}^{n}$ a sense-preserving spherical isometry such that $h_{z}(z)=0[\mathbf{V}, 12.2]$. It follows from (1.1) that for $r \in(0,1)$

$$
h_{z} Q(z, r)=Q(0, r)=B^{n}(w(r)) ; \quad w(r)=r / \sqrt{1-r^{2}},
$$

for all $z \in \bar{R}^{n}$. We shall identify $\bar{R}^{n}$ with the subset $\left\{x \in R^{n+1}: x_{n+1}=0\right\} \cup\{\infty\}$ of $\bar{R}^{n+1}$. Each Möbius transformation $f: \bar{R}^{n} \rightarrow \bar{R}^{n}$ has an extension $f^{*}: \bar{R}^{n+1} \rightarrow$ $\bar{R}^{n+1}$, the so-called Poincaré extension [B, p. 33], such that $f^{*}$ is a Möbius transformation with $f^{*} R_{+}^{n+1}=R_{+}^{n+1}$ and $f^{*} \mid \bar{R}^{n}=f$.

For a continuous mapping $f: \bar{R}^{n} \rightarrow \bar{R}^{n}$ and $t \in(0,1]$ we define

$$
\delta_{f}^{t}=\inf \left\{q(x, y): q(f(x), f(y))=t, x, y \in \bar{R}^{n}\right\} .
$$

If $\delta_{f}$ is the number in (1.3), then clearly $\delta_{f}=\delta_{f}^{1 / 2}$.

2.11. Lemma. Let $f: \bar{R}^{n} \rightarrow \bar{R}^{n}$ be a Möbius transformation, let $f^{*}: \bar{R}^{n+1} \rightarrow \bar{R}^{n+1}$ be its Poincaré extension, and let $\rho=\rho_{R_{+}^{n+1}}\left(e_{n+1}, f^{*}\left(e_{n+1}\right)\right)$. Then

$$
t e^{-\rho} \leqslant \delta_{f}^{t} \leqslant 2 t /\left(e^{\rho}+e^{-\rho}\right)
$$

for all $t \in(0,1]$.

Proof. The first inequality follows directly from Theorem 1.2 and from the definition (2.10). For the proof of the second inequality we note that the spherical distances change under $f$ in the same way as the euclidean distances change under 
$\pi \circ f \circ \pi^{-1}$, where $\pi$ is the stereographic projection (2.8). Then $\pi \circ f \circ \pi^{-1}$ is a Möbius self-mapping of the Riemann sphere. By performing an auxiliary translation followed by a stretching with factor 2 we may assume that $f$ is initially defined on $S^{n}$ and maps onto $S^{n} \subset R^{n+1}$. Formula (2.6) applied to the Poincaré extension of $f$ shows that $f=k \circ T_{a} \mid S^{n}$, where $k \in O(n+1), \rho_{B^{n+1}}(0, a)=\rho, T_{a}=p_{a} \circ \sigma_{a}$. Because $k$ and $p_{a}$ are euclidean isometries, it follows that the change of distances under $f$ is caused by the inversion $\sigma_{a}$. Fix $x_{t}, y_{t} \in S^{n}$ such that $\left|\sigma_{a}\left(x_{t}\right)-\sigma_{a}\left(y_{t}\right)\right|=2 t$ and

$$
\left|x_{t}-a^{*}\right|=\left|y_{t}-a^{*}\right| \leqslant\left|\sigma_{a}\left(x_{t}\right)-a^{*}\right|,
$$

where $a^{*}=a /|a|^{2}$ and such that the vectors $x_{t}-a^{*}, a, y_{t}-a^{*}$ are coplanar. Denote $u_{1}=\left|x_{t}-a^{*}\right|$ and $u_{2}=\left|\sigma_{a}\left(x_{t}\right)-a^{*}\right|$. By elementary geometry, $u_{1} u_{2}=$ $|a|^{-2}-1$. The properties of similar triangles yield

$$
\left|x_{t}-y_{t}\right|=2 u_{1} t / u_{2}=2 t\left(|a|^{-2}-1\right) / u_{2}^{2} \text {. }
$$

From the choice of $x_{t}$ it follows that $u_{2}^{2}=1+|a|^{-2}+2|a|^{-1} \sqrt{1-t^{2}}$ and hence

$$
\left|x_{t}-y_{t}\right| \leqslant \frac{2\left(|a|^{-2}-1\right) t}{1+|a|^{-2}}=\frac{4 t}{e^{\rho}+e^{-\rho}}
$$

because $\rho=\log ((1+|a|) /(1-|a|))$.

2.12. CoROllary. If $\delta_{f}$ is as in (1.3) and $f$ is as in Lemma 2.11, then

$$
e^{-\rho} / 2 \leqslant \delta_{f} \leqslant 1 /\left(e^{\rho}+e^{-\rho}\right), \quad \operatorname{Lip}(f) \leqslant 1 / \delta_{f} \leqslant 2 \operatorname{Lip}(f) .
$$

Proof. The proof follows from Lemma 2.11 and the fact that $\operatorname{Lip}(f)=e^{\rho}$ (cf. 1.2).

\section{The main results.}

Proof OF TheOREM 1.4(1). Let $h_{z}: \bar{R}^{n} \rightarrow \bar{R}^{n}$ be a spherical isometry as in (2.9) with $h_{z}(z)=0$. Because the inequality $1.4(1)$ is invariant under spherical isometries, we may as well assume $x=0, f(x)=0$, by considering $g=h_{f(x)} \circ f \circ h_{x}^{-1}$ (note that $\left.\delta_{f}=\delta_{g}\right)$. By (2.9) and the definition of $\delta_{f}$ we see that $f$ maps $B^{n}\left(w\left(\delta_{f}\right)\right)$ into $B^{n}(1 / \sqrt{3})$. By the quasiconformal version of the Schwarz lemma $[\mathbf{G 3}, \mathbf{V}, 18.2 ; \mathbf{V u 2}$, 3.3] we obtain for $x, y \in \bar{R}^{n}$ with $q(x, y)<\delta_{f}$,

$$
\begin{aligned}
\sqrt{3} q(f(x), f(y)) & \leqslant \frac{w(q(f(x), f(y)))}{w(1 / 2)} \\
& \leqslant \lambda_{n}\left(\frac{w(q(x, y))}{w\left(\delta_{f}\right)}\right)^{\alpha} \leqslant \lambda_{n}\left(\frac{q(x, y)}{\delta_{f}}\right)^{\alpha},
\end{aligned}
$$

where $\lambda_{n}$ is a positive constant depending only on $n$ and the function $w$ is as in (2.9). Because $4 \leqslant \lambda_{n}<e^{n}$ (cf. [GO, p. 61, (3.3)]), the above inequality holds trivially for $q(x, y) \geqslant \delta_{f}$, and the proof is thereby completed.

Proof of Theorem 1.5. We may assume $x=0=f(x)$. Fix $r \in(0, d(0, \partial G) / 2)$ and choose $x_{r}, y_{r} \in S^{n-1}(x, r)$ such that $\left|f\left(x_{r}\right)-f(x)\right|=L(x, f, r)$ and $\mid f\left(y_{r}\right)-$ $f(x) \mid=l(x, f, r)$. Let $\Gamma^{\prime}=\Delta\left(\left[0, f\left(y_{r}\right)\right],\left[f\left(x_{r}\right), \infty\right) ; f G\right)$ and $\Gamma=f^{-1} \Gamma^{\prime}$. It follows 
from (2.1) and Lemma 2.3(1) that

$$
M(\Gamma) \leqslant K M\left(\Gamma^{\prime}\right) \leqslant K \tau\left(\left(\left|f\left(x_{r}\right)\right| /\left|f\left(y_{r}\right)\right|\right)-1\right)
$$

and from Lemma 2.3(2) that

$$
M(\Gamma) \geqslant \tau(1)-2 \omega_{n-1}\left(\log \frac{d(0, \partial G)}{r}\right)^{1-n} .
$$

Letting $r \rightarrow 0$ yields $\tau(1) / K \leqslant \tau(H(0, f)-1)$. The inequality (1.6) follows, since $\tau$ is strictly decreasing.

3.1. Corollary. Let $f: R^{n} \rightarrow R^{n}$ be a K-quasiconformal mapping with $f(0)=0$. Then

$$
|f(x)| \leqslant c(n, K)|f(y)|
$$

for $|x| \leqslant|y|$, where $c(n, K)$ is as defined in (1.6).

Proof. The proof is similar to the above proof.

3.2. REMARK. With small changes the proof of Theorem 1.5 and Theorem 3.2 in [MRV] yield a similar upper bourd for the linear dilatation of a quasiregular mapping: if $f: G \rightarrow R^{n}$ is quasiregular and $x \in G$, then

$$
H(x, f) \leqslant c\left(n, i(x, f) K_{0}(f)\right),
$$

where $i(x, f)$ is the local topological index of $f$ at $x$ (see [MRV, p. 11]) and $K_{0}(f)$ is the outer dilatation of $f$ (see [MRV, p. 14]). The inequality (3.3) improves the bounds of Yu. G. Reshetnyak [R, Theorem 1, p. 1312] and O. Martio, S. Rickman and J. Väisälä [MRV, Theorem 4.5].

Denote $\gamma(s)=\operatorname{cap} R_{G}(s)$. In view of (2.2) the constant $c(n, K)$ in (1.6) can also be written as

$$
c(n, K)=\left[\gamma^{-1}(\gamma(\sqrt{2}) / K)\right]^{2}=\left[\gamma^{-1}\left(2^{n-1} \tau(1) / K\right)\right]^{2} .
$$

In particular, $c(n, 1)=2$. It is easy to see that (cf. e.g. [ $\mathbf{V u 2}, 2.16])$

$$
\begin{aligned}
& \gamma(s) \leqslant \omega_{n-1}\left(\log \left(s+\sqrt{s^{2}-1}\right)\right)^{1-n}, \\
& \gamma^{-1}(u) \leqslant \cosh \left[\left(\omega_{n-1} / u\right)^{1 /(n-1)}\right],
\end{aligned}
$$

where $\omega_{n-1}$ is the area of $S^{n-1}$. Now (3.4) and (3.5) give

$$
c(n, K) \leqslant \cosh ^{2}\left[\left(\frac{K \omega_{n-1}}{\gamma(\sqrt{2})}\right)^{1 /(n-1)}\right]=\cosh ^{2}\left[\frac{1}{2}\left(\frac{K \omega_{n-1}}{\tau(1)}\right)^{1 /(n-1)}\right]
$$

A. Mori [M, p. 61] proved Theorem 1.5 for $n=2$ with the constant $e^{\pi K}$ and an $n$-dimensional version of his result due to F. W. Gehring [G1, Lemma 8, p. 371] yields Theorem 1.5 for $n \geqslant 2$ with the constant

$$
d(n, K)=\exp \left[\left(\frac{K \omega_{n-1}}{\tau(1)}\right)^{1 /(n-1)}\right], \quad d(2, K)=e^{\pi K}
$$

It follows from (3.6) that $c(n, K)$ is strictly smaller than $d(n, K)$. 
For $n=2$ the best possible constant $\lambda(K)$ in Theorem 1.5,

$$
\lambda(K)=\frac{1}{16} e^{\pi K}-\frac{1}{2}+\delta(K) ; \quad \delta(K) \in\left(0,2 e^{-\pi K}\right),
$$

was found by $\mathbf{O}$. Lehto, K. I. Virtanen and J. Väisälä [LVV, p. 12]. We are now going to compare the numerical values of $c(2, K)$ and $\lambda(K)$. Making use of [ $\mathbf{L V}, \mathbf{p}$. 65] we observe that (3.5) can be improved for $n=2$ as follows (here $\gamma_{2}(s)$ is $\gamma(s)$ with $n=2$ ):

$$
\begin{aligned}
& \gamma_{2}(s) \leqslant 2 \pi / \log \left[s\left(1+\sqrt[4]{1-s^{-2}}\right)^{2}\right] \leqslant 2 \pi / \log \left(s+3 \sqrt{s^{2}-1}\right), \\
& \gamma_{2}^{-1}(u) \leqslant\left(3 \sqrt{\beta^{2}+8}-\beta\right) / 8 ; \quad \beta=\exp (2 \pi / u) .
\end{aligned}
$$

By (3.4) and (3.9) we obtain because $\gamma_{2}(\sqrt{2})=4[\mathbf{L V}$, p. 63, (2.8)]

$$
c(2, K) \leqslant\left(\left(3 \sqrt{\beta^{2}+8}-\beta\right) / 8\right)^{2} ; \quad \beta=\exp (\pi K / 2) .
$$

It follows from (3.8) and (3.10) that $c(2, K) / \lambda(K) \rightarrow 1$ as $K \rightarrow \infty$, and that for all $K \geqslant 1$,

$$
\begin{aligned}
& c(2, K) \leqslant\left(c^{2} / 64\right) \exp (\pi K)<\exp (\pi K) / 10, \\
& c=-1+3 \sqrt{1+8 e^{-\pi}}=2.480144 \ldots
\end{aligned}
$$

The proof in [LVV] was based on the Teichmüller theory, while the proof of Theorem 1.5 was a straightforward application of the modulus method. The crucial novel feature in its proof is the application of F. W. Gehring's new modulus inequality in Lemma 2.3(1) above.

By a theorem of Tukia and Väisälä [TV] for $n \geqslant 2$ and $K \geqslant 1$ there exists a number $K^{*}$ depending only on $n$ and $K$ such that each $K$-quasiconformal mapping $f: \bar{R}^{n} \rightarrow \bar{R}^{n}$ has a $K^{*}$-quasiconformal extension $f^{*}: \bar{R}^{n+1} \rightarrow \bar{R}^{n+1}$ such that $f^{*} \mid \partial R_{+}^{n+1}=f$ and $f^{*} R_{+}^{n+1}=R_{+}^{n+1}$. We are now going to prove the second part of Theorem 1.4 .

3.12. Lemma. Let $f$ and $f^{*}$ be as above and let $\delta_{f}$ be the number defined in (1.3). Then $\delta_{f}>0$ and there exist strictly increasing functions $\beta_{j}:[0, \infty) \rightarrow[0, \infty), j=1,2$, depending only on $n$ and $K$ such that

$$
\beta_{1}(\rho) \leqslant 1 / \delta_{f} \leqslant \beta_{2}(\rho),
$$

where $\rho=\rho_{R_{+}^{n+1}}\left(e_{n+1}, f^{*}\left(e_{n+1}\right)\right)$.

Proof. By performing an auxiliary Möbius transformation $h$ of $\bar{R}^{n+1}$ mapping $R_{+}^{n+1}$ onto $B^{n+1}$ and $e_{n+1}$ onto 0 , we may assume that $f: S^{n} \rightarrow S^{n}$ initially. Note that $2 q(x, y)=|h(x)-h(y)|$ for all $x, y \in \bar{R}^{n}$.

We shall first prove the left inequality. Let $f^{*}(0)=r e, e \in S^{n-1}, r>0$. Then $(1+r) /(1-r)=e^{\rho}$. Fix $y_{0} \in S^{n}$ such that $\left|y_{0}+e\right|=1$ and let $J^{\prime}$ be the shorter arc of a circle with radius one in $S^{n}$ connecting $-e$ with $y_{0}$. Then $J=f^{-1} J^{\prime}$ is an arc on $S^{n}$ with $d(J) \geqslant 2 \delta_{f}$. Let $A$ be a continuum connecting 0 with $S^{n}$. From a symmetry property of the modulus [G2, Lemma 1] and from some well-known inequalities (see [Vu1, 1.10, 1.11 or $\mathbf{V}$, pp. 30-40]) it follows that

$$
M\left(\Delta\left(A: J ; \bar{B}^{n+1}\right)\right) \geqslant 2^{-1} 3^{-n-4}(\log 2) c_{n+1} \delta_{f}=a_{n} \delta_{f}
$$


where $a_{n}$ is a positive number depending only on $n$. It follows from [V. 7.5] that

$$
M\left(\Delta\left([r e, e], J^{\prime}\right)\right) \leqslant \omega_{n}\left(\log \frac{\sqrt{3}}{1-r}\right)^{-n}=\omega_{n}\left(\log \frac{\sqrt{3}}{2}\left(1+e^{\rho}\right)\right)^{-n}
$$

Applying (3.13) with $A=f^{*-1}[r e, e]$ we obtain by (2.1)

$$
a_{n} \delta_{f} \leqslant \omega_{n}\left(\log \frac{\sqrt{3}}{2}\left(1+e^{\rho}\right)\right)^{-n} K^{*},
$$

from which the left inequality of the lemma follows.

For the proof of the right inequality we fix $x, y \in S^{n}$ such that $|x-y|=2 \delta_{f}$ and $|f(x)-f(y)|=1$. Such points exist by the definition of $\delta_{f}$. Let $J$ be the shorter circular arc of radius one in $S^{n}$ connecting $x$ with $y$, and let $z$ be the midpoint of $J$. If $|x-z|<1$, then we obtain by [V, 7.5]

$$
M(\Delta(J,[0,-z])) \leqslant \omega_{n}\left(\log \frac{1}{|x-z|}\right)^{-n} \leqslant \omega_{n}\left(\log \frac{\sqrt{3}}{2 \delta_{f}}\right)^{-n}
$$

and if $|x-z| \in[1, \sqrt{2}]$, then $\delta_{f} \geqslant \sqrt{3} / 2$ and we obtain

$$
M(\Delta(J,[0,-z])) \leqslant \omega_{n}(\log \sqrt{5})^{-n} \leqslant \frac{2 \delta_{f}}{\sqrt{3}} \omega_{n}(\log \sqrt{5})^{-n} .
$$

We shall next prove a lower bound for $M\left(f^{*} \Delta(J,[0,-z])\right)$. Let $f^{*}(0)=s e, e \in S^{n}$. Because $1-s=2 /\left(1+e^{\rho}\right)$ and $d(f J) \geqslant 1-s$, we get by [Vu1, 1.10, 1.11 and G2, Lemma 1],

$$
\begin{aligned}
M\left(\Delta\left(f J, f^{*}[0,-z]\right)\right) & \geqslant 2^{-1} 3^{-n-4} c_{n+1}(\log 2) 2 /\left(1+e^{\rho}\right) \\
& =d_{n} /\left(1+e^{\rho}\right) .
\end{aligned}
$$

Because $f$ is quasiconformal, the right inequality of the lemma follows from (3.15), $(3.15)^{\prime},(3.16)$ and (2.1).

\section{REFERENCES}

[A] L. V. Ahlfors, Möbius transformations in several variables, School of Mathematics, Univ. of Minnesota, 1981.

[AVV] G. D. Anderson, M. K. Vamanamurthy and M. Vuorinen, Dimension-free, quasiconformal distortion in $n$-space (to appear).

[B] A. F. Beardon, The geometry of discrete groups, Graduate Texts in Math., Vol. 91, Springer-Verlag, Berlin and New York, 1983.

[G1] F. W. Gehring, Rings and quasiconformal mappings in space, Trans. Amer. Math. Soc. 103 (1962), 353-393.

[G2] __ A remark on domains quasiconformally equivalent to a ball, Ann. Acad. Sci. Fenn. Ser. A I Math. 2 (1976), 147-155.

[G3] _ Quasiconformal mappings. Complex Analysis and its Applications (Lectures, Internat. Sem. Triest, 1975), Vol. II, Internat. Atomic Energy Agency, Vienna, 1976, pp. 213-268.

[GO] F. W. Gehring and B. G. Osgood, Uniform domains and the quasihyperbolic metric, J. Analyse Math. 36 (1979), 50-74.

[LV] O. Lehto and K. I. Virtanen, Quasikonforme Abbildungen, Grundlehren der Math. Wissenschaften, Vol. 126, Springer-Verlag, Berlin and New York, 1965.

[LVV] O. Lehto, K. I. Virtanen and J. Väisälä, Contributions to the distortion theory of quasiconformal mappings, Ann. Acad. Sci. Fenn. Ser. A I Math. 273 (1959), 1-14. 
[MRV] O. Martio, S. Rickman and J. Väisälä, Definitions for quasiregular mappings, Ann. Acad. Sci. Fenn. Ser. A I Math. 448 (1969), 1-40.

[M] A. Mori, On quasiconformality and pseudo-analyticity, Trans. Amer. Math. Soc. 84 (1957), 56-77.

[R] Yu. G. Reshetnyak, Local structure of mappings with bounded distortion, Sibirsk. Mat. Zh. 10 (1969), 1311-1333. (Russian)

[TV] P. Tukia and J. Väisälä, Quasiconformal extension from dimension $n$ to $n+1$, Ann. of Math. (2) 115 (1982), 331-348.

[V] J. Väisälä, Lectures on n-dimensional quasiconformal mappings, Lecture Notes in Math., Vol. 229, Springer-Verlag, Berlin and New York, 1971.

[Vu1] M. Vuorinen, On the existence of angular limits of n-dimensional quasiconformal mappings, Ark. Mat. 18 (1980), 157-180.

[Vu2] _. Conformal invariants and quasiregular mappings, J. Analyse Math. (to appear).

Department of Mathematics, University of Helsinki, Helsinki, Finland 